OPEN ACCESS

Edited by:

Patricia Cogram,

Fraunhofer Chile Research, Chile

Reviewed by:

Isidre Ferrer,

University of Barcelona, Spain Christian Gonzalez-Billault,

University of Chile, Chile

*Correspondence:

Eduardo M. Castaño ecastano@/eloir.org.ar

tThese authors have contributed equally to this work.

Received: 26 July 2016 Accepted: 28 February 2017

Published: 14 March 2017

Citation:

Belfiori-Carrasco LF, Marcora MS Bocai NI, Ceriani MF, Morelli L and Castaño EM (2017) A Novel Genetic

Screen Identifies Modifiers of Age-Dependent Amyloid $\beta$ Toxicity in the Drosophila Brain.

Front. Aging Neurosci. 9:61. doi: 10.3389/fnagi.2017.00061

\section{A Novel Genetic Screen Identifies Modifiers of Age-Dependent Amyloid $\beta$ Toxicity in the Drosophila Brain}

\author{
Lautaro F. Belfiori-Carrasco ${ }^{1 \dagger}$, María S. Marcora ${ }^{1 \dagger}$, Nadia I. Bocai ${ }^{1}$, M. Fernanda Ceriani ${ }^{2}$, \\ Laura Morelli ${ }^{1}$ and Eduardo M. Castaño ${ }^{1 *}$ \\ 'Laboratorio de Amiloidosis y Neurodegeneración, Fundación Instituto Leloir-Instituto de Investigaciones Bioquímicas de \\ Buenos Aires, Consejo Nacional de Investigaciones Cientificas y Técnicas (CONICET), Buenos Aires, Argentina, ${ }^{2}$ Laboratorio \\ de Genética del Comportamiento, Fundación Instituto Leloir-Instituto de Investigaciones Bioquímicas de Buenos Aires, \\ Consejo Nacional de Investigaciones Cientificas y Técnicas (CONICET), Buenos Aires, Argentina
}

The accumulation of amyloid $\beta$ peptide $(A \beta)$ in the brain of Alzheimer's disease (AD) patients begins many years before clinical onset. Such process has been proposed to be pathogenic through the toxicity of $A \beta$ soluble oligomers leading to synaptic dysfunction, phospho-tau aggregation and neuronal loss. Yet, a massive accumulation of $A \beta$ can be found in approximately $30 \%$ of aged individuals with preserved cognitive function. Therefore, within the frame of the "amyloid hypothesis", compensatory mechanisms and/or additional neurotoxic or protective factors need to be considered and investigated. Here we describe a modifier genetic screen in Drosophila designed to identify genes that modulate toxicity of $A \beta 42$ in the CNS. The expression of $A \beta 42$ led to its accumulation in the brain and a moderate impairment of negative geotaxis at 18 days post-eclosion (d.p.e) as compared with genetic or parental controls. These flies were mated with a collection of lines carrying chromosomal deletions and negative geotaxis was assessed at 5 and 18 d.p.e. Our screen is the first to take into account all of the following features, relevant to sporadic AD: (1) pan-neuronal expression of wild-type A 342 ; (2) a quantifiable complex behavior; (3) $A \beta$ neurotoxicity associated with progressive accumulation of the peptide; and (4) improvement or worsening of climbing ability only evident in aged animals. One hundred and ninety-nine deficiency (Df) lines accounting for $\sim 6300$ genes were analyzed. Six lines, including the deletion of 52 Drosophila genes with human orthologs, significantly modified $A \beta 42$ neurotoxicity in 18-day-old flies. So far, we have validated CG11796 and identified CG17249 as a strong candidate (whose human orthologs are HPD and PRCC, respectively) by using RNAi or mutant hemizygous lines. PRCC encodes proline-rich protein PRCC (ppPRCC) of unknown function associated with papillary renal cell carcinoma. HPD encodes 4-hydroxyphenylpyruvate dioxygenase (HPPD), a key enzyme in tyrosine degradation whose Df causes autosomal recessive Tyrosinemia type 3, characterized by mental retardation. Interestingly, lines with a partial Df of HPD ortholog showed increased

\footnotetext{
Abbreviations: $\mathrm{AD}$, Alzheimer's disease; $\mathrm{A} \beta$, amlyoid $\beta$ peptide; $\mathrm{A} \beta 42$, amyloid $\beta$ peptide 1-42; $\mathrm{CK}$, creatine kinases Df, deficiency; DIOPT, Drosophila RNAi Screen Center Integrative Ortholog Prediction Tool; dpe, days post-eclosion; G4, Gal4; H\&E, hematoxylin-eosin; HPPD, 4-hydroxy-phenylpyruvate dioxygenase; PBS, phosphate buffered saline; Ppil2, peptidylprolyl isomerase-like 2; ppPRCC, proline-rich protein PRCC; qRT-PCR, quantitative real-time PCR; RING, Rapid Iterative Negative Geotaxis; RM, repeated measures; SDS-PAGE, sodium dodecyl sulfate-polyacrylamide gel electrophoresis; ThS, thioflavine S; UAS, upstream activating sequence.
} 
intraneuronal accumulation of $A \beta 42$ that coincided with geotaxis impairment. These previously undetected modifiers of $A \beta 42$ neurotoxicity in Drosophila warrant further study to validate their possible role and significance in the pathogenesis of sporadic AD.

Keywords: amyloid $\beta$, Alzheimer's disease, neurodegeneration, genetic screen, Drosophila, dementia

\section{INTRODUCTION}

Alzheimer's disease (AD) is the most prevalent form of dementia in the aged population worldwide and its impact is steadily growing due to the extension of life expectancy (Cacace et al., 2016; Scheltens et al., 2016). More than 95\% of AD cases are sporadic, with age and the epsilon 4 allele of the apolipoprotein $\mathrm{E}$ gene as the major risk factors. Rare familial forms are associated with mutations in the amyloid precursor protein and presenilin 1-2 genes (Campion et al., 1995; Newman et al., 2007; Kandimalla et al., 2011, 2012; De Strooper and Karran, 2016).

$\mathrm{AD}$ brain is characterized by a pervasive synaptic loss and the accumulation of protein aggregates mostly composed of $\mathrm{A} \beta 42$ and microtubule-associated protein tau. Oligomeric species of $A \beta 42$ have been proposed as early pathogenic molecules by inducing mitochondrial and endoplasmic reticulum stress, an increase in reactive oxygen species formation and action potential abnormalities (Karran et al., 2011; De Strooper and Karran, 2016). AD tau is excessively phosphorylated and aggregates intracellularly leading to microtubule instability and organelle failure (Khan and Bloom, 2016). However, the accumulation of $A \beta$ and phospho-tau is not sufficient for the development of AD. Large autopsy series show that about $30 \%-40 \%$ of individuals can sustain a normal or nearly normal cognitive function at a very old age despite extensive $\mathrm{A} \beta$ and phospho-tau pathology (Bennett et al., 2006; Maarouf et al., 2011; Perez-Nievas et al., 2013). Several hypothesis have been put forward to explain such clinico-pathological dissociation, including differences in "cognitive/brain reserve" or the presence of compensatory mechanisms at a functional or molecular level (Maarouf et al., 2011; Steffener and Stern, 2012). In this context, the search for novel genetic and epigenetic factors that partake in neurotoxicity mechanisms related to $\mathrm{A} \beta$ is of key importance for understanding the disease process.

Drosophila is widely used for genetic screens applied to study the molecular bases of neurodegenerative disorders including AD (Crowther et al., 2005; Moloney et al., 2010; Lenz et al., 2013; Prüßing et al., 2013; Shulman et al., 2014; Fernandez-Funez et al., 2015; Liu et al., 2015). Major advantages of this animal model include a complex CNS, the fact that about $70 \%$ of human genetic diseases have a Drosophila genetic counterpart (Jackson, 2008; Bouleau and Tricoire, 2015; Lim et al., 2016) and the availability of large collections of mutant and transgenic lines.

Forward genetic screens in Drosophila have been used to identify modifiers of $\mathrm{A} \beta$ neurotoxicity. Cao et al. (2008) used a collection of transgenic lines carrying directionally inserted $\mathrm{P}$ elements and screened for enhancers or suppressors of a rough eye phenotype induced by $A \beta 42$. In this way, they identified candidate genes involved in cellular processes such as transcription regulation, proteolysis in the secretory pathway and cholesterol metabolism (Finelli et al., 2004; Cao et al., 2008). By screening a collection of chromosomal deletions, the same group found that the toll-NFKB pathway enhanced both $\mathrm{A} \beta$-induced rough eye and a negative effect upon life span (Tan et al., 2008). Rival et al. (2009) screened 3000 lines carrying $\mathrm{P}$ element inserts for modifiers of a shorter life span induced by the "Arctic" variant of $A \beta 42$ (A $\beta E 22 G$ ) associated with familial AD. Notably, they found that genes associated with redox or antioxidant activities were strong modifiers of $A \beta E 22 \mathrm{G}$ neurotoxicity (Rival et al., 2009). By inducing misexpression of genes involved in specific developmental pathways, several modifiers of $\mathrm{A} \beta 42$ toxicity upon photoreceptors have been described (Moran et al., 2013). In addition to the eye phenotype and life span, the gravitaxis behavior (negative geotaxis) can be used for genetic screening. This test provides easily quantifiable data, explores a complex behavior of the Drosophila CNS and allows a rapid assessment of age-dependent $\mathrm{A} \beta$ toxicity. Recently, Liu et al. (2015) developed an automatic device for the Rapid Iterative Negative Geotaxis (RING) assay and screened a collection of chromosomal deletions to find modifiers of A $\beta E 22 \mathrm{G}$ neurotoxicity upon the giant fiber system neurons (Gargano et al., 2005; Liu et al., 2015).

The aim of the present study was to develop a modifier screen designed to study the effect of chromosomal deletions upon neuronal toxicity mediated by pan-neural expression of wild-type $\mathrm{A} \beta 42$ in the CNS (the major isoform that accumulates in the brain of sporadic $\mathrm{AD}$ patients). Fly lines with defined genomic deletions were found to exert a dominant effect under the presence of $A \beta 42$. Deficiency (Df) lines that significantly enhanced age-dependent $A \beta 42$ toxicity included CG17249 and CG11796 whose human orthologs are $P R C C$ and $H P D$, respectively. $P R C C$ encodes proline-rich protein PRCC (ppPRCC), a protein of unknown function associated with renal cell carcinomas. HPD encodes 4-hydroxyphenylpyruvate dioxygenase (HPPD), a key enzyme in tyrosine degradation.

\section{MATERIALS AND METHODS}

\section{Fly Stocks}

Flies were raised at $25^{\circ} \mathrm{C}$ in a standard corn meal with a light:dark cycle of $12 \mathrm{~h}: 12 \mathrm{~h}$. The line expressing A $\beta 1-42$ fused with the rat pre-proenkephalin signal peptide was kindly provided by Dr. Mary Konsolaki (Rutgers University). The upstream activating sequence (UAS)-A $\beta 42$ construct is inserted in the 2 nd chromosome. Lines $w^{1118}$ \#5905 (+), elav ${ }^{\mathrm{c} 155}$ [Gal4] \#458 (G4), lines from the Df kit and the mutants for CG11796 \#51528 and 
CG17249 \#16098 were obtained from Bloomington Drosophila Stock Center (NIH P0OD018537). The CG11796 RNAi line \#103482 was obtained from VDRC Stock Center. The elav [Gal4]; [UAS] $A \beta 42 /$ Cyo line $(G 4>A \beta 42)$ was generated for the screen.

\section{RING Assay}

Groups of $30-40$ male flies were raised at $25^{\circ} \mathrm{C}$ in 4 -inch glass vials with food replacement every 2-3 days. The geotaxis behavior was tested using the RING assay as described (Gargano et al., 2005). The day before the test, 10 flies were shortly anesthetized with $\mathrm{CO}_{2}$ and placed into a fresh vial. They were let to recover overnight at $25^{\circ} \mathrm{C}$, transferred to clear glass vials and placed them in the negative geotaxis device. The device was tapped three times in rapid succession to initiate the response and climbing was recorded for $10 \mathrm{~s}$. The climbed distance in $\mathrm{cm}$ was measured for each fly and the average height from five technical replicates per genotype was calculated using the Scion Image software.

\section{SDS-PAGE and Western Blots}

Forty heads from 5 to 18 -day-old flies were homogenized in $60 \mu \mathrm{l}$ of RIPA buffer, pH 7.4, containing 1\% SDS, 5 mM EDTA, $5 \mathrm{mM}$ EGTA, $1 \mathrm{mM}$ PMSF, $0.5 \mu \mathrm{g} / \mathrm{ml}$ leupeptin, $0.5 \mu \mathrm{g} / \mathrm{ml}$ aprotinin, $1 \mathrm{mg} / \mathrm{ml}$ pepstatin and $50 \mathrm{mM} \mathrm{NaF}$. Homogenates were centrifuged at $10,000 \times \mathrm{g}$ for $1 \mathrm{~h}$ at $4^{\circ} \mathrm{C}$. Twenty $\mu \mathrm{l}$ of the supernatant containing $\sim 150 \mu \mathrm{g}$ of total proteins, were resolved by sodium dodecyl sulfate-polyacrylamide gel electrophoresis (SDS-PAGE) in a $12.5 \%$ Tris-tricine gel. After transfer to polyvinylidene fluoride membranes, proteins were analyzed by Western blot. $A \beta 42$ was detected with anti- $A \beta$ monoclonal 6E10 (Biolegend Co.) used at 1:1000. Actin was detected with rabbit polyclonal anti-actin (Sigma) at 1:1000. After washing with PBS-T, membranes were incubated anti-rabbit or anti-mouse horseradish peroxidase-labeled IgGs (Dako, Denmark) at 1:10000. Immunoreactivity was visualized by chemiluminescence with ECL Prime (GE Bioscience, Piscataway, NJ, USA) and scanned with an Image Quant LAS 4000 apparatus (GE Bioscience, Piscataway, NJ, USA). For relative quantitation, optical densities from each lane were obtained and analyzed with the ImageJ software. Synthetic A $\beta 1-42$ was obtained from American Peptide Co.

\section{Inmunohistochemistry and Thioflavin S Staining}

Adult heads were fixed with $4 \%$ paraformaldehyde in phosphate buffered saline (PBS) for $45 \mathrm{~min}$ at room temperature (RT). Fly brains were dissected in PBS containing $0.1 \%$ Triton X-100 (PT). Brains were blocked in 10\% normal goat serum for $1 \mathrm{~h}$ in PT and incubated with antibody $6 \mathrm{E} 10$ at $4^{\circ} \mathrm{C}$ overnight. After incubation with Cy3-labeled anti-mouse antibody (Jackson InmunoResearch, West Grove, PA, USA) for $2 \mathrm{~h}$ at RT, brain tissue was stained with DAPI, washed with PBS and mounted in PBS containing 80\% glycerol. For amyloid fibril staining, brains were incubated in 50\% ethanol containing $1 \%$ thioflavine S (ThS; Sigma, St.Louis, MO, USA) overnight at $4^{\circ} \mathrm{C}$. Samples were washed with PBS containing 50\% ethanol and mounted in $80 \%$ glycerol. Brain samples from a transgenic mouse carrying the "Swedish" mutation of amyloid precursor protein (Tg2576) were used as positive controls. Images were captured with a Zeiss LSM 510 Meta Confocal microscope.

\section{Histology and Vacuolization Assessment}

Fly heads were fixed overnight in Carnoy solution (60\% ethanol, $30 \%$ chloroform, $10 \%$ acetic acid) at $4^{\circ} \mathrm{C}$ and dehydrated in increasing concentrations of ethanol. Then, they were treated with butanol:ethanol (1:1), butanol:toluene (1:1) and toluene $30 \mathrm{~min}$ each, and finally soaked in toluene:paraffin (1:1) for $30 \mathrm{~min}$ at $65^{\circ} \mathrm{C}$. After a 2 -h incubation at $65^{\circ} \mathrm{C}$ in pure paraffin, heads were embedded and cut in $8 \mu \mathrm{m}$ serial frontal sections. After H\&E staining, images were captured using an OLYMPUS $\mathrm{B} \times 50$ Microscope and analyzed with the Image J software. Brain tissue loss was quantified as described (Sarantseva et al., 2009). The area occupied by vacuoles with a diameter of at least $3 \mu \mathrm{m}$ was divided by the total area of the section and expressed as percentage of area loss. At least eight brains per genotype were analyzed.

\section{Genetic Screen}

To perform the genetic screen, the G4 > A $\beta 42$ line was mated with Df lines from the Bloomington Df kit (Cook et al., 2012; Cook, 2016) to generate elav $v^{\mathrm{cl} 55}$ [Gal4]; [UAS] A $342 /+>$ Df/+ $(\mathrm{G} 4>\mathrm{A} \beta 42 / \mathrm{Df})$. The experimental design consisted of three stages (Figure 1). In stage I, G4 > A $\beta 42 / D$ f lines were analyzed at 5 and 18 days post-eclosion (d.p.e) to find a modified phenotype as compared to G4 > A $\beta 42$. Genetic controls included G4 $>+$ and $+>\mathrm{A} \beta 42$. Those Df lines that showed a difference of at least $50 \%$ in negative geotaxis only at 18 d.p.e in a single biological experiment were selected. In stage II, each chromosomal deletion; elav ${ }^{\mathrm{c} 155}$ [Gal4]; Df/+ (G4 > Df) was assessed to rule out that it did not affect negative geotaxis in the absence of $\mathrm{A} \beta 42$ expression. Three independent biological experiments were performed comparing G4 > A $\beta 42$ with $\mathrm{G} 4>\mathrm{A} \beta 42 / \mathrm{Df}$ to select the Df lines that reached statistical significance. Deleted genes were queried for the identification of human orthologs with expression in the adult CNS (see below). If the deletion was large and included more than 10 human orthologs, overlapping deletions were analyzed as in stage II to reduce the number of candidates. Deletions with less than 10 human orthologs were selected for analysis with RNAi or mutant lines in stage III.

\section{Bioinformatic Analysis of Deficiency Lines}

Genomic deletions were queried in Bloomington Stock web page $^{1}$. The corresponding gene list was obtained from FlyBase ${ }^{2}$ (Attrill et al., 2016) using the GBrowse function and the Hit List tool. Each gene was searched for its human ortholog with the highest weighted score using the Drosophila RNAi Screen Center (DRSC) Integrative Ortholog Prediction Tool (DIOPT)

\footnotetext{
${ }^{1}$ http://www.flystocks.bio.indiana.edu/Browse/df/dfkit.php

${ }^{2}$ http://www.flybase.org
} 


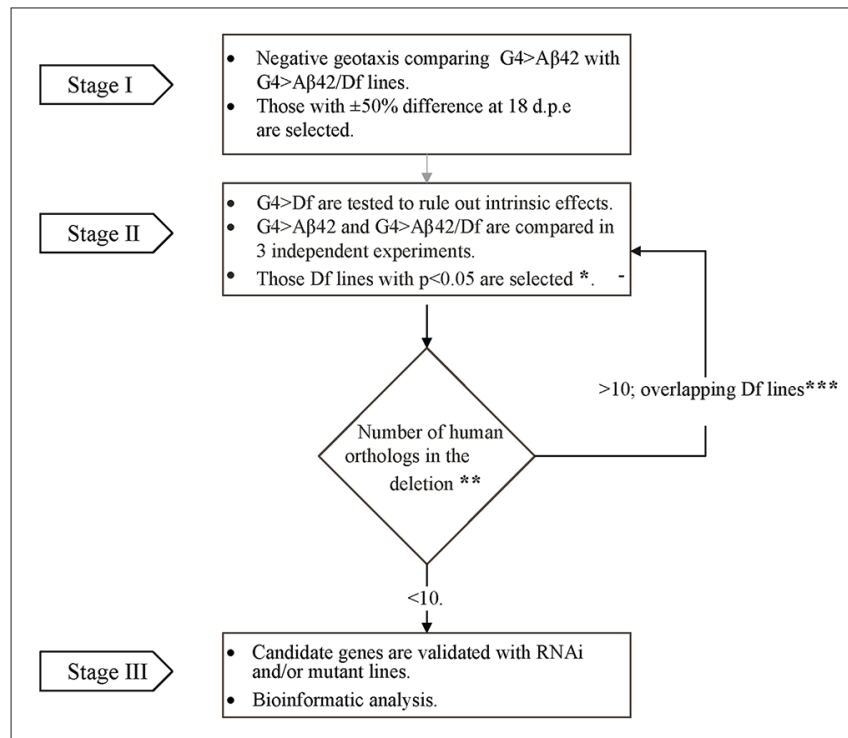

FIGURE 1 | Flow chart illustrating the overall strategy and steps of the modifier genetic screen. In stage I, G4 > amyloid $\beta$ peptide 1-42 (A $\beta 42)$ line was compared to each of the G4 > A $\beta 42 /$ Deficiency (Df) lines in a single negative geotaxis experiment. *In stage II, those G4 > AB/Df lines selected in stage I were examined in three independent biological experiments for statistical significance at 18 days post eclosion (d.p.e), (one-way ANOVA followed by least significant difference (LSD) Fisher's test $p<0.05)$. **Human orthologs were defined as those with the highest score according to Drosophila RNAi Screen Center (DRSC) integrative ortholog prediction tool (DIOPT). Depending on the number of deleted orthologs ( $>$ or $\leq 10$ ), Df lines were selected for stage III or back to stage II analysis with overlapping deletions to narrow down the number of candidates $\left({ }^{* * *}\right.$ Overlapping deletions were compared in three independent biological experiments).

from the DRSC ${ }^{3}$. Fly gene expression was searched in NCBI web page ${ }^{4}$ and the RNA-seq Profile provided by FlyBase on the gene query subtitle expression data ${ }^{5}$. Gene products, their known functions, patterns of expression in humans, proteinprotein interactions and association with human diseases were obtained from UNIPROT ${ }^{6}$, Genecards ${ }^{7}$ and OMIM $^{8}$ databases.

\section{Preparation of cDNA Samples and Quantitative Real-Time PCR}

RNA from 35 fly heads was extracted with the TriZol reagent (Invitrogen) according to manufacturer's instructions. cDNA was generated from $3 \mu \mathrm{g}$ of RNA, previously treated with DNAse (Promega) using the SuperScript III system (Invitrogen). SYBR-Green quantitative real-time PCR (qRT-PCR) was performed using KAPA SYBR_FAST Universal 2X qPCR Master Mix. Reactions were run in a Stratagene Mx3005P cycler (Agilent Technologies) and analyzed by the calibration curve method. For CG11796 primers 5'AAAGGAACCAAACCTGAA GC 3'

${ }^{3} \mathrm{http} / / /$ www.flyrnai.org/cgi-bin/DRSC_orthologs.pl

${ }^{4}$ http://www.ncbi.nlm.nih.gov/gene

${ }^{5}$ http://flybase.org/reports/FBgn0036992.html

${ }^{6} \mathrm{http}: / /$ www.uniprot.org/

${ }^{7} \mathrm{http} / / /$ www.genecards.org/

${ }^{8} \mathrm{http}: / /$ www.omim.org/ (forward) and 5'ATCCCTGATAGCCAAGTGGT 3' (reverse) were used. RPL32 was amplified for normalization using the following primers: 5'ATGCTAAGCTGTCGCACA AATG 3' (forward) and 5'GTTCGATCCGTAACCGATGT 3' (reverse).

\section{Statistical Analysis}

Results are presented as the mean \pm SEM of at least three independent biological experiments unless otherwise stated. Data were analyzed by repeated measures (RM) two-way ANOVA with post hoc Bonferroni's test, RM one-way ANOVA followed by Least Significant Difference (LSD) Fisher's test or Student's $t$ test using the Prism ${ }^{\circledR}$ Graphpad 6 software. Wilcoxon non-parametric test were used when indicated. The level of significance was set at $p<0.05$.

\section{RESULTS}

\section{G4 > Aß42 Line Shows a Moderate and Age-Dependent Toxic Phenotype}

A transgenic line with constitutive, pan-neuronal expression of $\mathrm{A} \beta 42$ maintained at $25^{\circ} \mathrm{C}$ was examined as a candidate for the screen. Western blots of fly head homogenates showed a $\sim 4.5 \mathrm{kDa}$ band consistent with detergent-soluble A $\beta 42$ correctly targeted and cleaved in the secretory pathway. A minor band consistent with SDS-resistant A $\beta 42$ oligomers was also seen. Between 5 and 18 d.p.e there was a robust 3-fold increase of A $\beta 42$ levels (Figures 2A,B). Negative geotaxis was not impaired in 5-day-old flies as compared with controls, strongly suggesting that there were no developmental effects upon the CNS due to $A \beta 42$ expression. In 18-day-old flies, a significant decrease in climbing ability $(\sim 50 \%)$ was apparent only in $\mathrm{A} \beta 42$ expressing animals as compared to genetic controls, G4>+ and $+>A \beta 42$ (Figure 2C). Microscopic examination of the brains of affected flies revealed very mild vacuolization and negative ThS staining (see below). Therefore, this line showed age-dependent $\mathrm{A} \beta 42$ accumulation and CNS neurotoxicity, and the magnitude of the functional decline was optimal for the search of enhancers and suppressors. In addition, the accretion of non-fibrillar A $\beta 42$ suggests that toxicity was induced by soluble oligomers, as proposed for AD. Taken together, these features and experimental conditions made this A 342 transgenic line highly suitable for a forward genetic screen.

\section{Identification of Df Lines that Modify Age-Dependent A $\beta 42$ Toxicity}

One hundred and ninety-nine lines with defined deletions from the 2nd, 3rd and 4th chromosomes, accounting for approximately 6300 genes, were tested in the first stage of the screen. Negative geotaxis of G4>A $\beta 42$ line was compared to $\mathrm{G} 4>\mathrm{A} \beta 42 / \mathrm{Df}$ lines at 5 and 18 d.p.e. Figure 3 shows actual examples of the three possible outcomes: Df 29667 had no modifying effect, Df 27917 worsened and Df 7681 rescued $A \beta 42$-induced climbing dysfunction. At this stage, $73 \mathrm{G} 4>\mathrm{A} \beta 42 / \mathrm{Df}$ lines showed a difference in climbing ability of at least $50 \%$ when compared to $\mathrm{G} 4>\mathrm{A} \beta 42$ and 


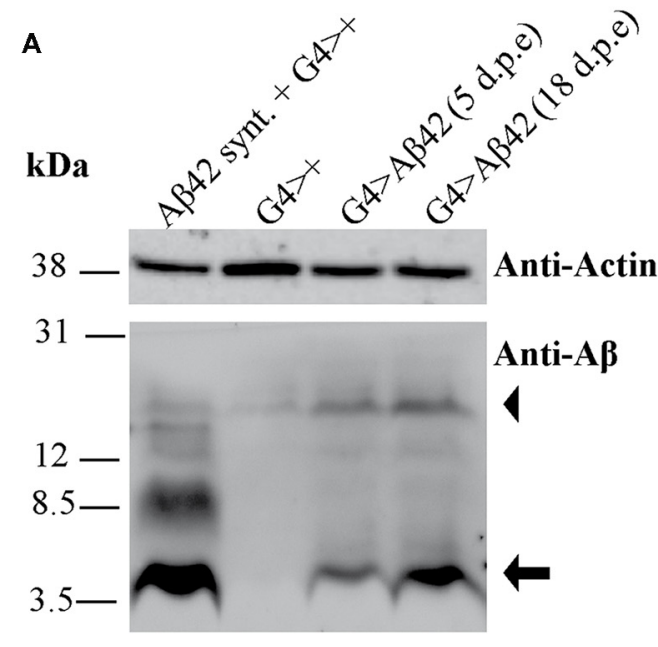

B

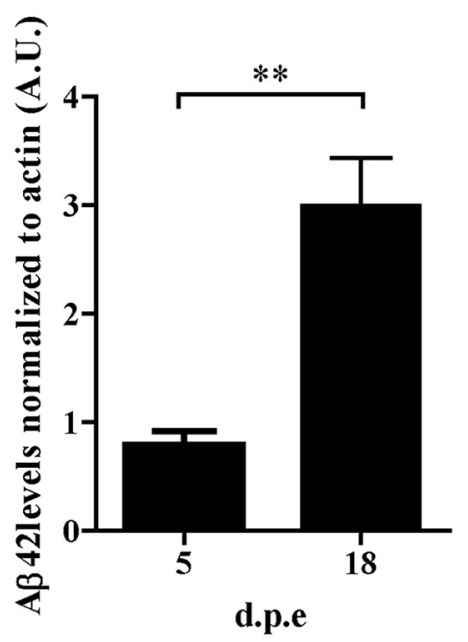

C

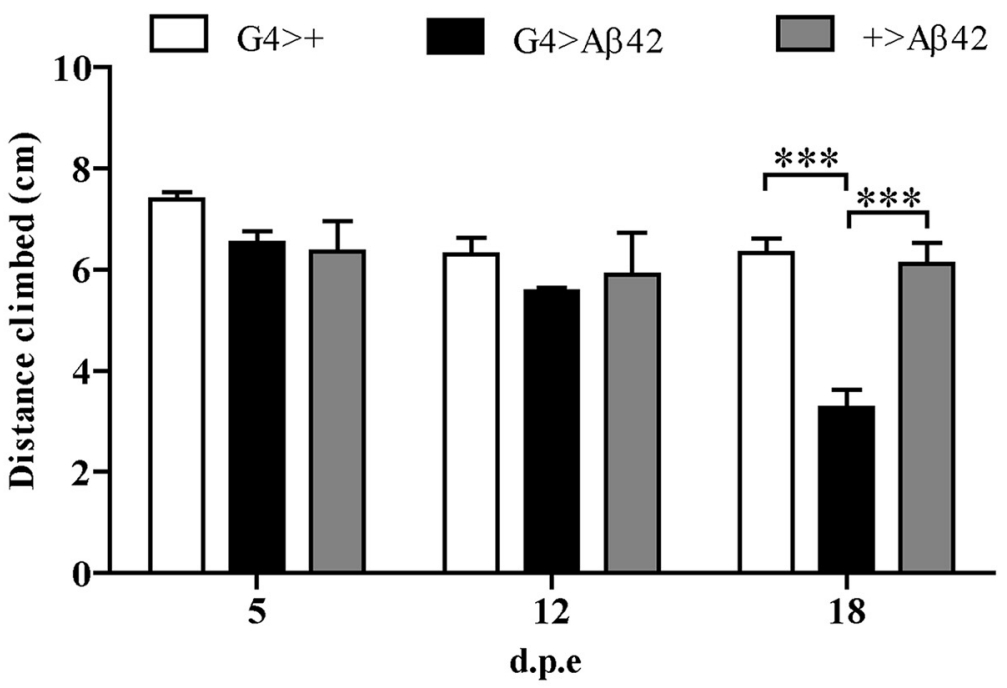

FIGURE 2 | (A) Representative Western blot of fly brain homogenates at 5 and 18 d.p.e showing A 42 expression detected with anti-A $\beta$ monoclonal antibody $6 E 10$. The $4.5 \mathrm{kDa}$ band (arrow) indicates A 42 correctly processed in the secretory pathway. The arrowhead indicates a band consistent with sodium dodecyl sulfate

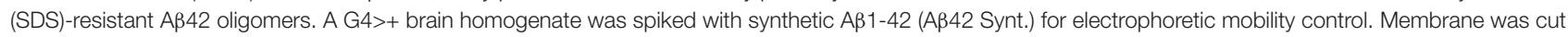
above the $31 \mathrm{kDa}$ marker and probed with anti-actin for normalization. (B) Quantification of A 42 levels relative to actin in arbitrary units (A.U.). Bars represent the mean \pm SEM from three independent experiments; ${ }^{* *} p<0.01$ (Student's $t$-test). (C) Pan-neuronal A A42-expressing flies (G4 > A 42 ) showed climbing impairment at 18 d.p.e as compared with genetic controls (G4>+ and +>AB42). Bars represent the mean \pm SEM from at least three independent biological experiments; ${ }^{* * *} p<0.001$ (repeated measures [RM] two-way ANOVA followed by Bonferroni's post hoc test).

such differences were only seen in aged animals. These lines were selected and analyzed in stage II and six lines met statistical criteria to be considered as positive hits. Df lines 24392, 27369, 27372, 27404 and 27917 worsened negative geotaxis while line 7681 reduced $A \beta 42$ toxicity to a full rescue of the phenotype (Figure 4). In the absence of $\mathrm{A} \beta 42$ expression, Df lines showed no intrinsic effect and none of the enhancer Df lines induced climbing impairment in G4 > A 42 line at 5 d.p.e, ruling out a possible acceleration of $\mathrm{A} \beta 42$ toxicity (not shown). Within these six Df lines, 36 Drosophila genes with human orthologs remain to be tested to identify enhancers and 14 genes to pin point suppressors of $\mathrm{A} \beta 42$ neurotoxicity. Interestingly, 14 out of 15 enhancer and six out of seven suppressor Df lines described in a previous screen based on negative geotaxis (Liu et al., 2015) were selected in stage I of our screen but did not reach statistical significance in stage II and were not further analyzed. 


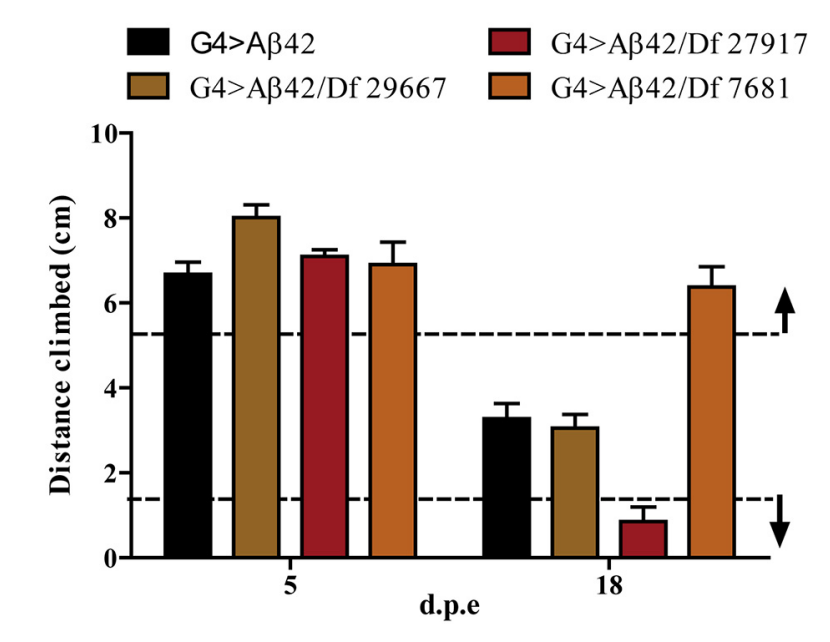

FIGURE 3 | Negative geotaxis assay of G4 > A 342 compared to G4 > A $\mathbf{\beta} 42 /$ Df at $\mathbf{5}$ and $\mathbf{1 8}$ d.p.e. The graphic shows examples of the three possible outcomes according to the quantitative criterion of at least a $50 \%$ difference in negative geotaxis (dashed lines): Df 29667 had no modifier effect, Df 27917 worsened and Df 7681 improved the climbing ability of A 342 -expressing flies at 18 d.p.e. Bars represent the mean \pm SEM from a single biological experiment (five technical repeats) and therefore, at this stage of the screen, no statistical analyses were performed.

\section{Specific Reduction of CG11796 Expression Enhances A $\beta 42$ Toxicity}

Thus far, three out of the five enhancer Df lines that passed stage II have been partially analyzed in stage III. The enhancer Df line 27372 included the deletion of CG17249 whose human ortholog is PRCC. We used a line carrying a Piggy Bac transposon in the $3^{\prime}$ region of CG17249 to assess toxicity. A significant enhancement in $A \beta 42$ neurotoxicity was observed in mutant hemizygous flies (Figure 5A). Although unlikely, the 3 ' insertion may compromise the expression of neighboring genes and therefore, RNAi experiments are required to validate CG17249. Df lines 27917 and 27369 also worsened negative geotaxis in the presence of $A \beta 42$ and the overlapping chromosomal segment included CG11796 whose human ortholog is HPD encoding HPPD, a key enzyme involved in tyrosine catabolism. To determine if a reduced expression of CG11796 was capable of enhancing $A \beta 42$ toxicity, we used two independent approaches: a mutant line in which a $\mathrm{Mi}[\mathrm{Mic}]$ transposon was inserted in the CG11796 gene and a specific RNAi with pan-neuronal expression using the elav promoter. These lines had no impairment in negative geotaxis as compared with control flies despite the reduction of CG11796 mRNA. Yet, in the presence of pan-neuronal A $\beta 42$ expression, CG11796 downregulation in both the RNAi and mutant lines induced a significant enhancement of $A \beta 42$ toxicity, similar to the overall effect of the chromosomal deletions detected at stages I-II of the screen (Figures 5B,C). The specificity of the RNAi was assessed by qRT-PCR from fly heads, which showed a strong reduction of CG11796 mRNA of approximately 85\% in G4 > CG11796 $6^{\mathrm{RNAi}}$ and $55 \%$ in CG1 $1796^{\text {Mut }}$, similar to the expected $~ 50 \%$ mRNA reduction in Df line 27917 (Figure 5D).

\section{Reduction of CG11796 Expression Promotes the Accumulation of Non-Fibrillar A $\beta 42$}

$\mathrm{A} \beta 42$ levels were analyzed in the brains of flies with partial Df of CG11796 at 18 days of age, when the toxic phenotype was detected. Confocal immunofluorescence showed extensive intraneuronal perinuclear $A \beta$ accumulation which was $\sim 2$-fold higher in both CG11796 hemizygous mutant and CG11796 ${ }^{\mathrm{RNAi}}$ as compared with flies expressing $\mathrm{A} \beta 42$ alone (Figure 6). Western blots of head homogenates showed a $70 \%-80 \%$ increase in the $\mathrm{A} \beta$ monomer band in CG11796 mutant and RNAi lines, consistent with the immunofluorescence results (Figure 7). The increment of $\mathrm{A} \beta$ abundance was not accompanied by ThS staining, indicating that a partial Df of CG11796 expression promoted the accumulation of non-fibrillar $A \beta$ species (Figure 8). Instead, the pattern of immunostaining and detergent solubility suggest the accretion of intraneuronal oligomeric $\mathrm{A} \beta$ which concurs with a higher neurotoxicity in CG11796 ${ }^{\text {Mut }}$ and CG11796 ${ }^{\mathrm{RNAi}}$ flies. To assess neurodegeneration further, the extent of vacuolization in the brain was determined for each genotype. As mentioned above, there was a mild though significant increase of vacuolization in flies expressing $A \beta 42$ as compared with their genetic controls. Yet, tissue loss did not increase in A $\beta 42$-transgenic flies expressing CG11796 mutant or RNAi (Figure 9). Together, these results strongly suggest that a partial Df of the HPD ortholog promotes the accumulation of toxic A 342 oligomers in the CNS leading to cellular dysfunction without histologically detectable neuronal loss.

\section{DISCUSSION}

The finding of proteins that modulate $A \beta$ neurotoxicity in animals with a complex CNS such as Drosophila may impact on $\mathrm{AD}$ research in several ways. First, by providing novel players in the cellular mechanisms by which $A \beta$ promotes synaptic dysfunction and neuronal death. Second, changes in the levels or activity of those proteins may be validated in human samples including post-mortem tissue and, more relevant, in biological fluids as potential biomarkers. Third, in the long range, it may open therapeutic strategies alternative to the current ones mostly aimed at $A \beta$ and tau. Previous modifier screens in the fly have yielded interesting candidates that modulate wild-type $A \beta$ toxicity in the eye, upon life span, or negative geotaxis induced by an aggressive $A \beta$ mutant (Cao et al., 2008; Tan et al., 2008; Rival et al., 2009; Liu et al., 2015). Our screen was designed to search for modifiers in a context of neurotoxicity more related to what may occur in sporadic $\mathrm{AD}$, including pan-neuronal expression of wild-type $A \beta 42$ and age-dependent accumulation with no detectable behavioral impairment in young animals. Moreover, the A 342 transgenic line had a rather mild phenotype at $\sim 3$ weeks of age, with little neuronal loss and the accumulation of detergent-soluble, non-fibrillar species of $\mathrm{A} \beta$, avoiding features that are found in late stages of $\mathrm{AD}$.

The discrepancies between our results and those reported by Liu et al. (2015) may be due to criteria for defining positive hits and the use in their study of $A \beta E 22 G$ driven to 
A
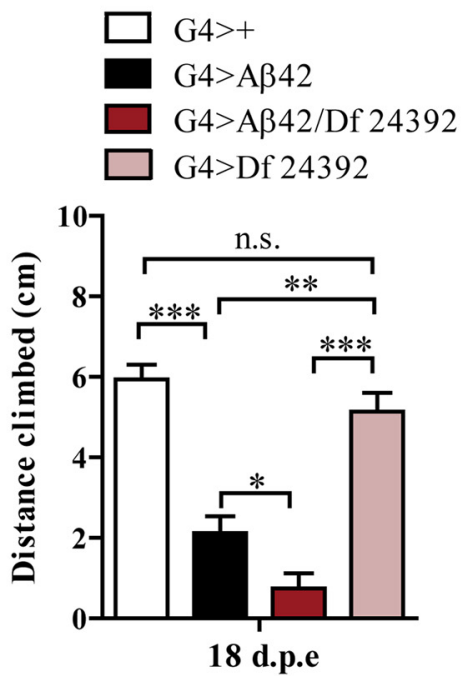

D
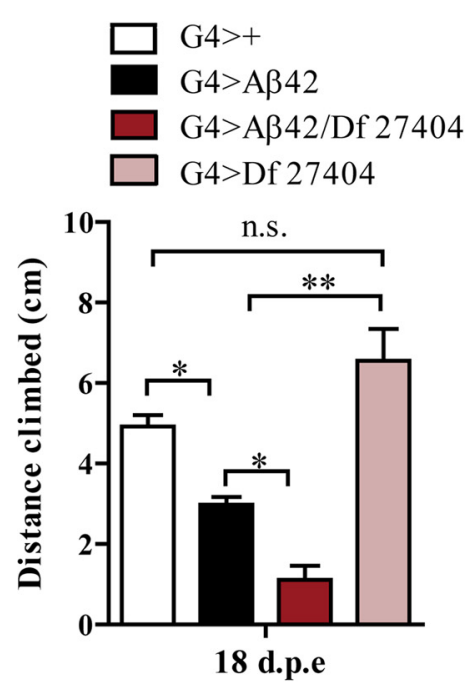

B
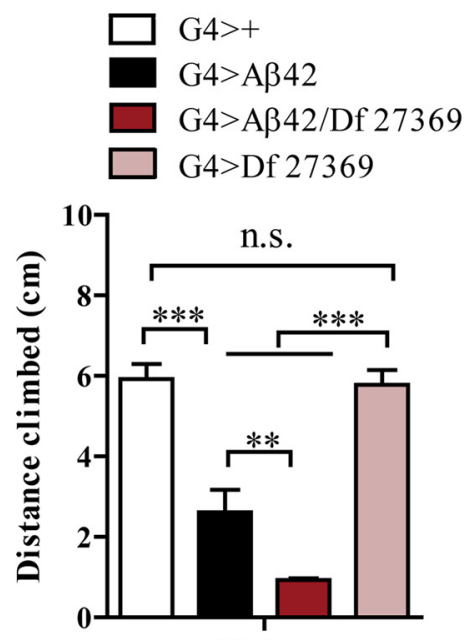

18 d.p.e

E
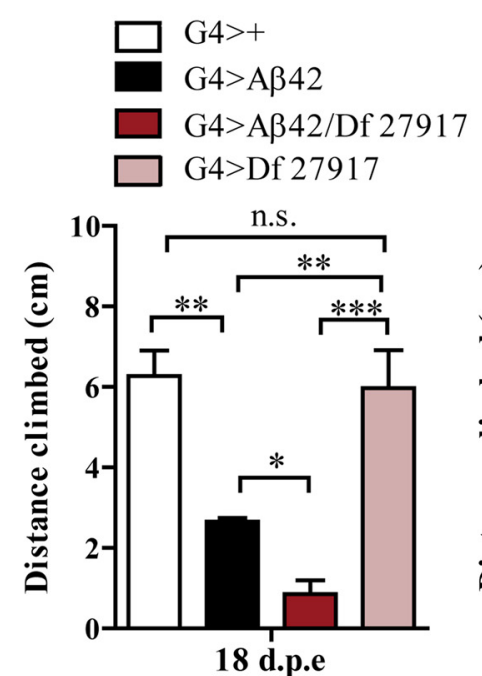

C
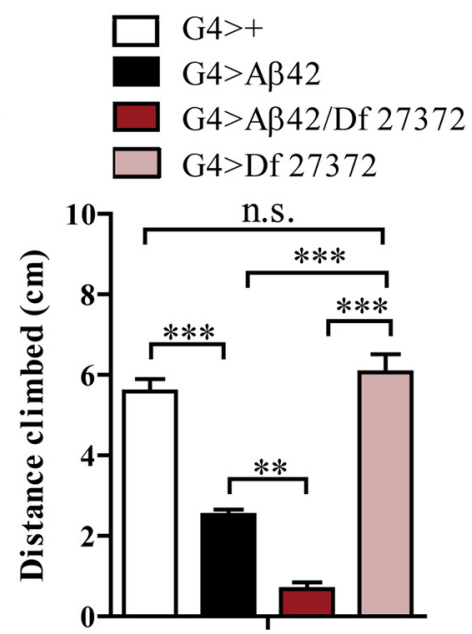

18 d.p.e

F
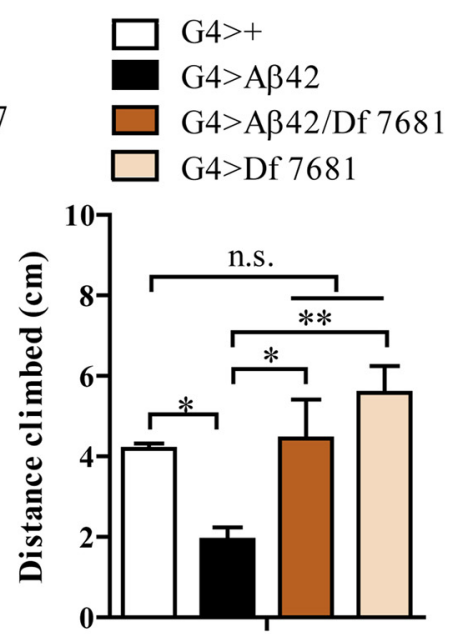

18 d.p.e

FIGURE 4 | Negative geotaxis assay of G4>+, G4 > A A42, G4 > A A42/Df and G4 > Df at 18 d.p.e. (A) Df 24392; (B) Df 27369; (C) Df 27372; (D) Df 27404; and (E) Df 27917, worsened the A 442 -induced phenotype. (F) Df 7681 improved the climbing ability of A 342 -expressing flies. Df lines had no effect in the absence of $\mathrm{A} \beta$ expression. Bars represent the mean \pm SEM from at least three independent biological experiments; ${ }^{*} p<0.5,{ }^{* *} p<0.01,{ }^{* * *} p<0.001$ (RM one-way ANOVA followed by LSD Fisher's test).

specific interneurons that relay to thoracic muscles instead of pan-neuronal wild-type $A \beta 42$. Noteworthy, in both studies Df line 7681 was a strong suppressor, suggesting that one or more genes in homozygosity within this deletion are necessary for $A \beta$ to impair geotaxis behavior, independent of $A \beta$ species and type of neurons involved.

A limitation of our study was its restriction to the effect of gene deletions and therefore, likely dependent on lower than physiological levels of the encoded proteins. Those genes that modulate $\mathrm{A} \beta 42$ toxicity through overexpression would be missed with our strategy.
So far, two genes have passed stage III of our screen whose human orthologs are PRCC and HPD. While PRCC requires a final validation step with RNAi, HPD was unambiguously identified. The function of ppPRCC is largely unknown although early studies suggest that it may have a role in pre-mRNA splicing (Skalsky et al., 2001). A search for ppPRCC proteinprotein interactions revealed association with peptidylprolyl isomerase-like 2 (Ppil2), a chaperone with putative ubiquitin ligase activity (Hatakeyama et al., 2001; Pushkarsky et al., 2005; Hegele et al., 2012). Thus, a possible role of a ppPRCC-Ppil-2 complex in protein folding, transport and degradation warrants 
A

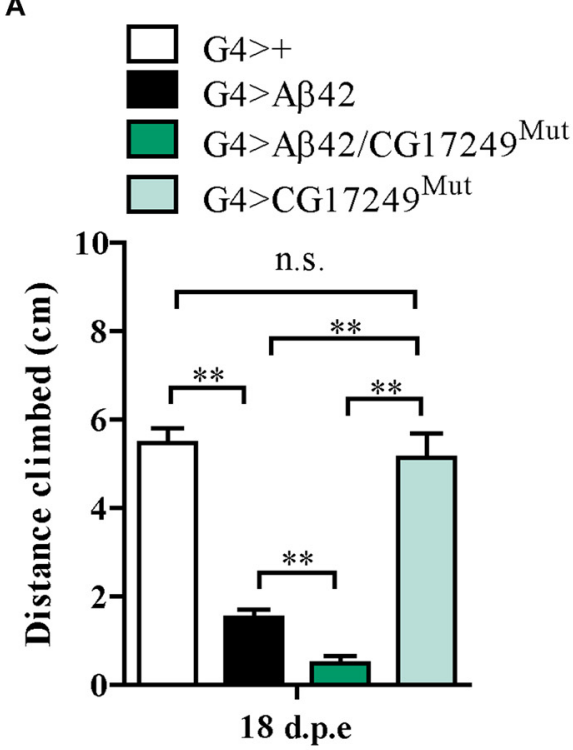

C

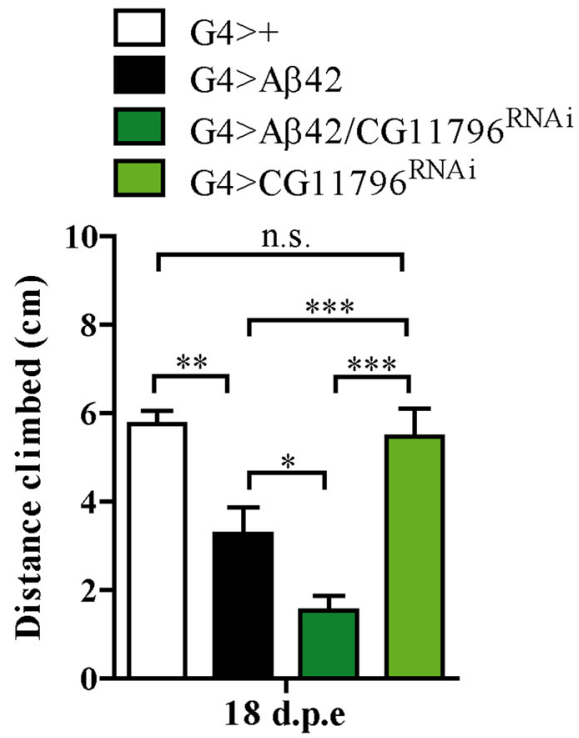

B

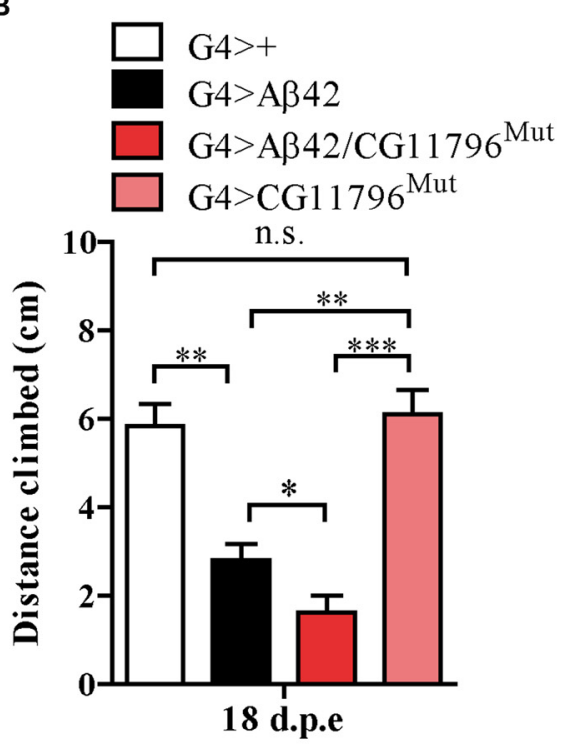

D

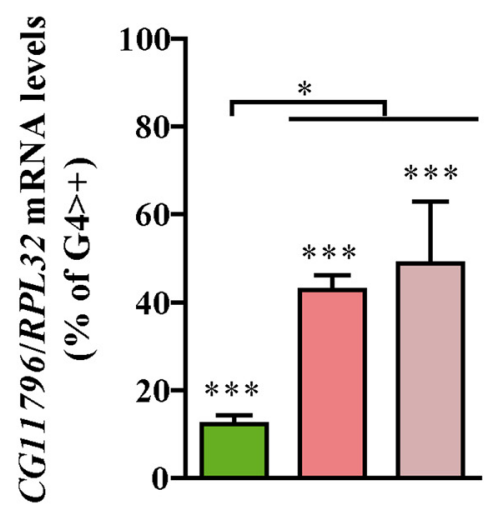

FIGURE 5 | Mutants and RNAi of candidate genes enhance A $\beta$ toxicity. (A) CG17249 hemizygous mutant (human ortholog, PRCC); (B) CG11796 hemizygous mutant (human ortholog, HPD) and (C) CG11796 RNAi. Mutant and RNAi lines had no effect in the absence of A 42 expression. Bars represent mean \pm SEM from at least three independent biological experiments; ${ }^{*} p<0.5 ;{ }^{* *} p<0.01 ;{ }^{* * *} p<0.001$ (one-way ANOVA followed by LSD Fisher's test). (D) Quantification of CG11796 endogenous mRNA showed a 40\%-50\% reduction in Df 27917 and CG11796 Mut lines, while for CG11796RNAi a 85\% reduction was observed. Brain samples were taken from 5 day-old flies and RPL32 mRNA was used for normalization in each quantitative real-time PCR (qRT-PCR) assay. ${ }^{* * *} p<0.001$ for Df 27917, CG11796 ${ }^{\text {Mut }}$ and CG11796 ${ }^{\text {RNAi }}$ as compared to G4>+. ${ }^{*} p<0.05$ (RM one-way ANOVA followed by LSD Fisher's test from three independent biological experiments).

further study in the context of $\mathrm{A} \beta$ neurotoxicity. $H P D$ encodes a highly conserved protein that catalyzes the conversion of 4-hydroxyphenylpyruvate to homogentisate, the second step in the tyrosine degradation pathway. Mutations in HPD cause the rare diseases Tyrosinemia type 3 and Hawkinsiuria. Tyrosinemia type 3 is autosomal recessive; patients show mental retardation and elevated levels of tyrosine and its derivatives in blood and urine due to HPPD Df (reviewed in Scott, 2006). Hawkinsinuria 

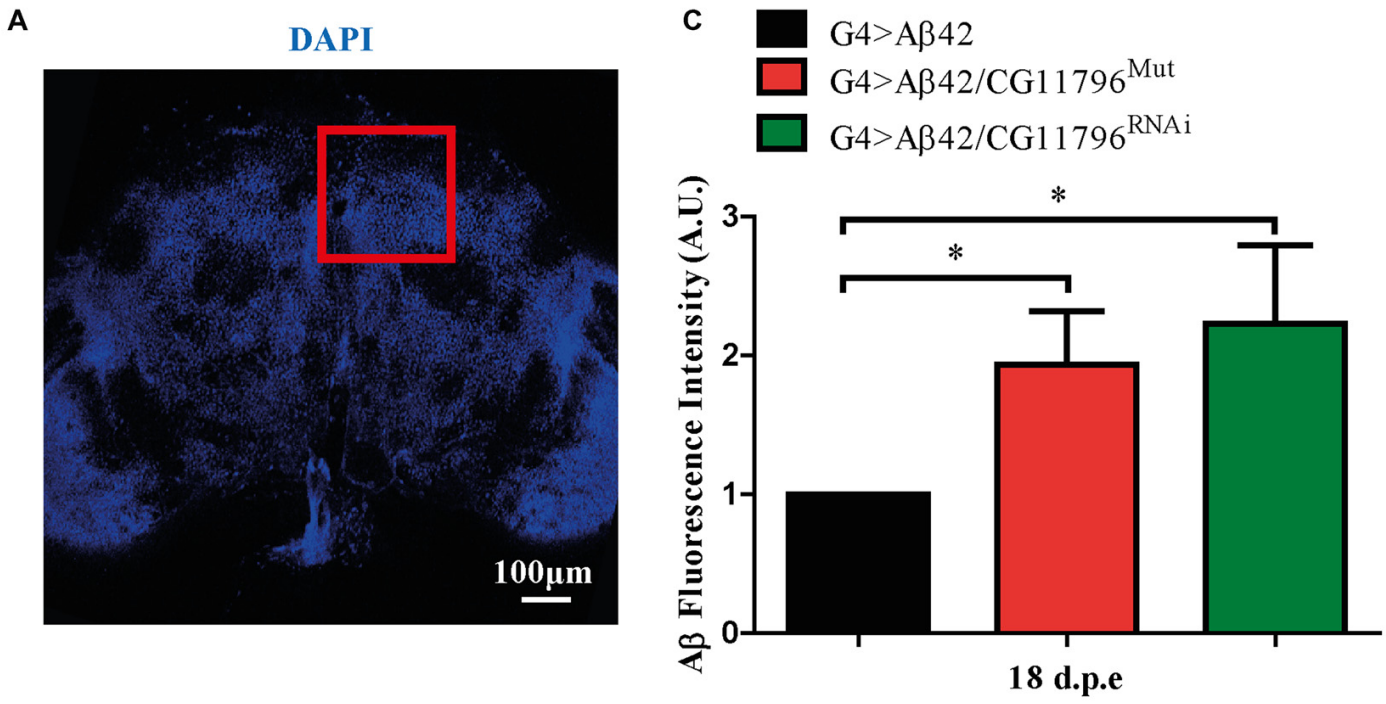

B
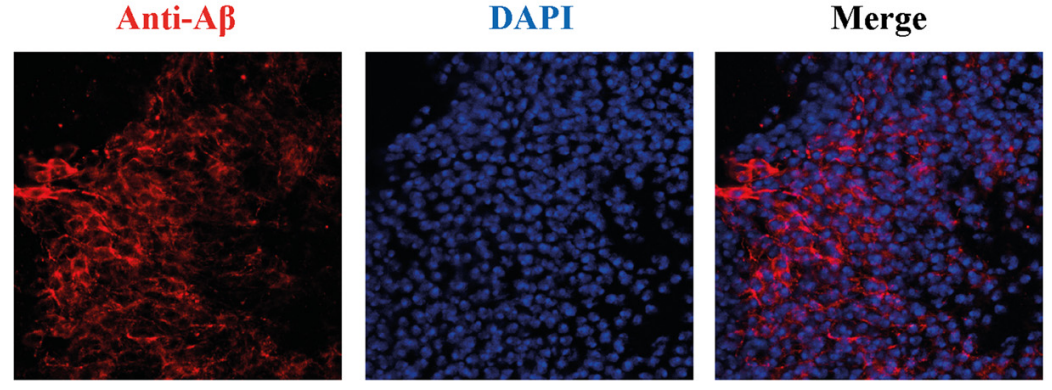

G4 $>$ Aß42/CG11796 ${ }^{\text {Mut }}$
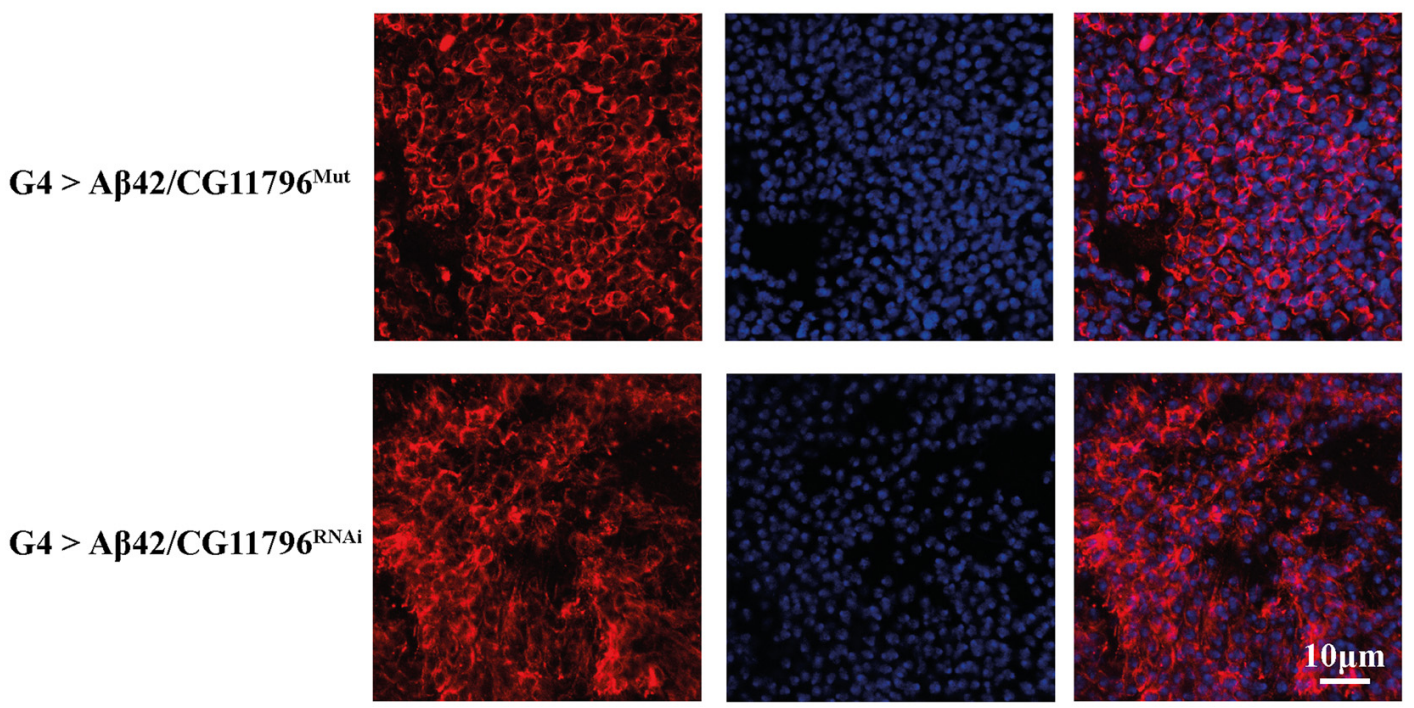

FIGURE 6 | Immunofluorescence of A $\beta$ deposits in the brains of transgenic lines. (A) Representative image of a brain section at low magnification stained with DAPI. The red square depicts the region used for quantification in each genotype. Scale bar $=100 \mu \mathrm{m}$. (B) Representative images of the selected region as in panel (A) showing from left to right: anti-A $\beta$, DAPI nuclear staining and the merge of both signals. Scale bar $=10 \mu \mathrm{m}$. Genotypes G4 >AB42,

G4 > AB42/CG11796 ${ }^{\text {Mut }}$ and G4 > AB42/CG11796 RNAi are shown. (C) Quantification of A fluorescence intensity normalized to G4 > AB42 in A.U. showing the increment induced by CG11796 mRNA reduction. Bars represent the mean-ratio \pm SEM of three independent experiments; ${ }^{*} p<0.05$ (Wilcoxon test). 
A

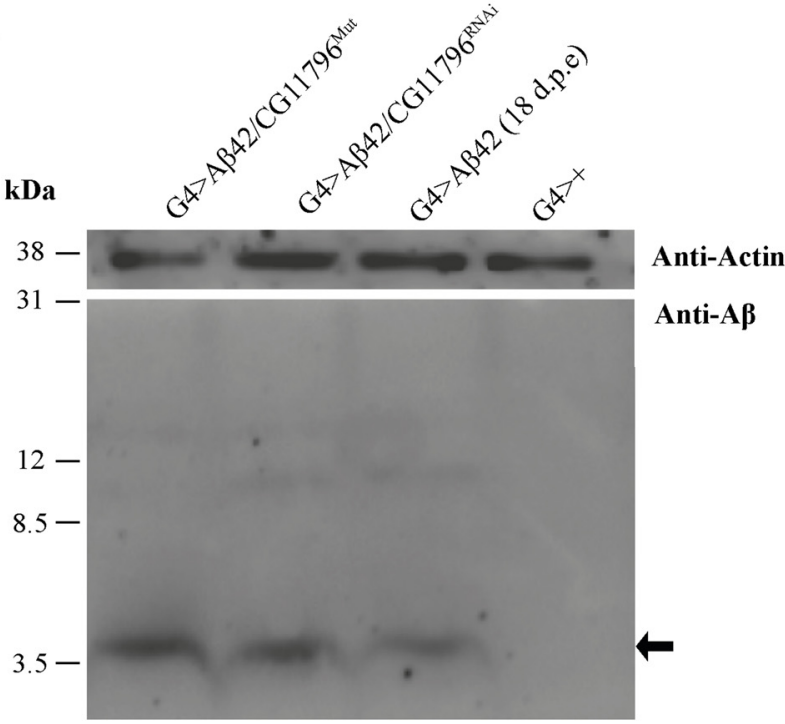

B

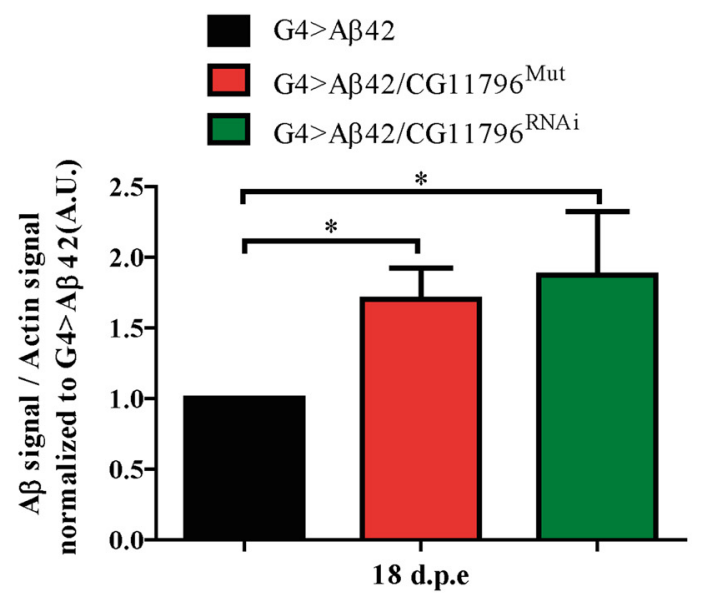

FIGURE 7 | Western blot of A $\beta$ accumulation in the brain of CG11796 RNAi and mutant lines. (A) Representative Western blot of fly brain homogenates in RIPA buffer at 18 d.p.e showing A 42 expression detected with monoclonal antibody $6 E 10$. The arrow indicates A 42 monomers. Membranes were cut above the $31 \mathrm{kDa}$ marker and probed with anti-actin for normalization. (B) Quantification of A $\beta 42$ levels relative to actin in A.U. normalized to G4 > A 42 showing the increase of A 42 in CG11796 mutant and RNAi lines. Bars represent the mean-ratio \pm SEM of three independent experiments; * $p<0.05$ (Wilcoxon test).

A

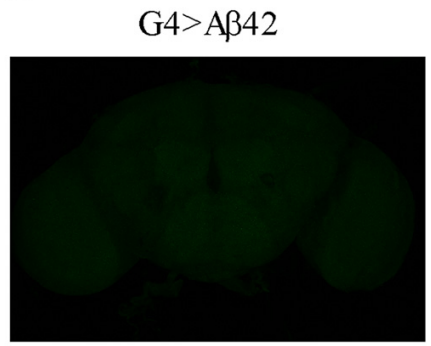

C G4>A $>42 / \mathrm{CG} 11796$

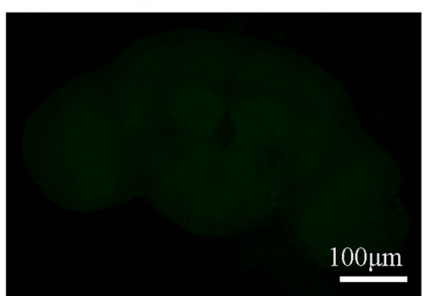

B

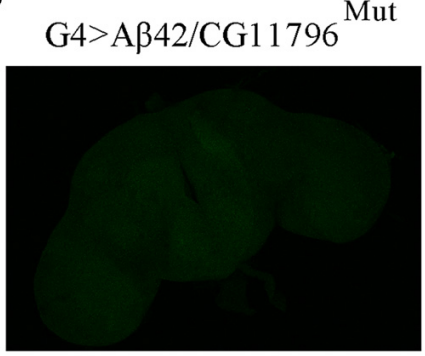

D

$\operatorname{Tg} 2576$ (mouse)

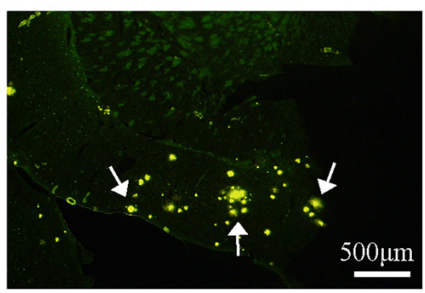

FIGURE 8 | Negative thioflavine S (ThS) staining of Aß42 transgenic flies brains. (A-C) Representative images of ThS staining of fly brains at 18 d.p.e from

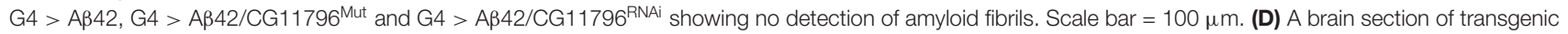
mouse Tg2576 showing ThS-positive plaques (arrows) is shown for comparison. Scale bar $=500 \mu \mathrm{m}$.

is autosomal dominant and characterized by metabolic acidosis and urinary excretion of "hawkinsin", a cyclic amino acid derived from quinolacetic acid produced by mutant HPPD (Brownlee et al., 2010). The mechanisms underlying mental retardation in Tyrosinemia are not known, yet an increase of acetylcholinesterase activity and energy metabolic impairment have been postulated (Ferreira et al., 2012, 2015). In addition, high tyrosine levels may reduce the activity of thiol-dependent 


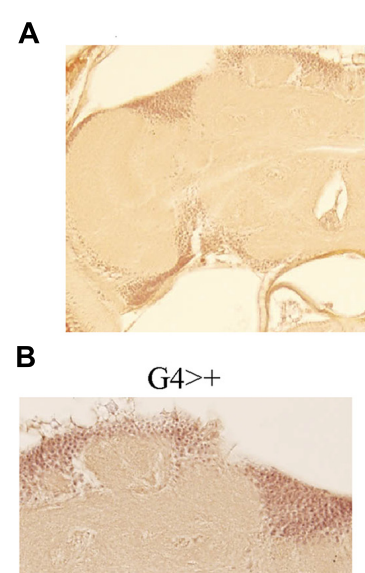

G4> CG11796

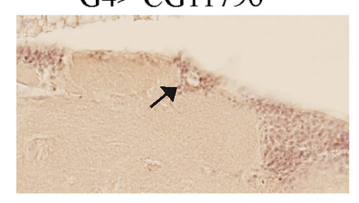

G4> CG11796

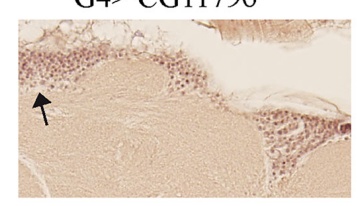

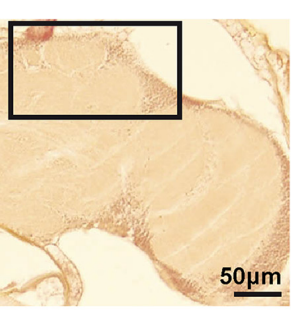
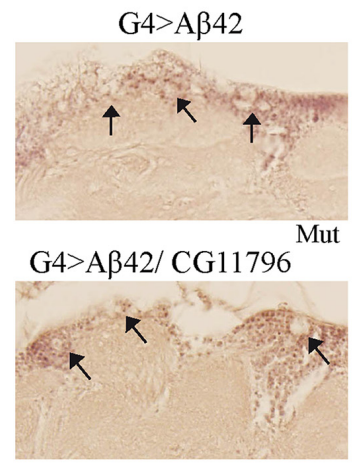

G4>A $342 /$ CG11796

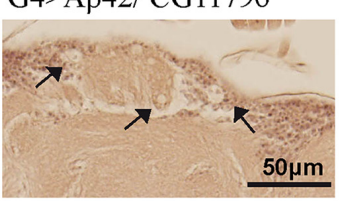

C
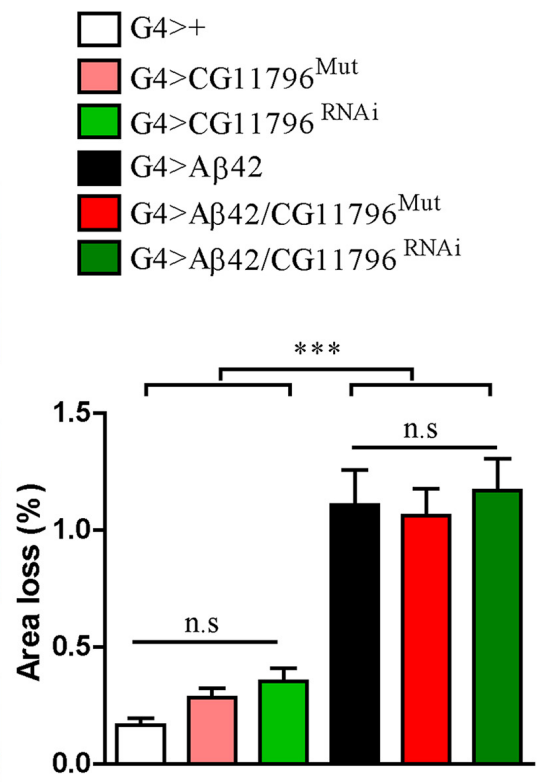

FIGURE 9 | Brain vacuolization in A $\beta$-expressing lines alone and in a background of CG11796 Df. (A) Representative whole brain section of G4>+ stained with H\&E used for tissue loss analysis by bright-field microscopy. The rectangle demarcates a typical area with a high number of neuronal bodies. (B) The region depicted in (A) is shown for each genotype. Arrows indicate vacuoles with a diameter of at least $3 \mu \mathrm{m}$. Scale bar $=50 \mu \mathrm{m}$. (C) Quantification of tissue loss in hemi-brains was calculated as the percentage of the section area occupied by vacuoles. Flies expressing A 442 showed increased vacuolization as compared to

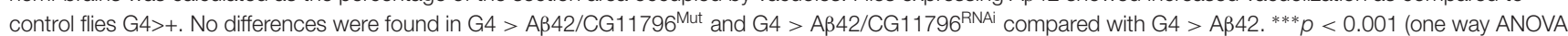
followed by Tukey's post hoc test).

creatine kinases (CK) leading to misbalance of a key ATP buffering and shuttling system (Wallimann et al., 2011; de Andrade et al., 2012). Interestingly, CK activity is reduced in AD brains as compared to age-matched controls and $A \beta$ induces a reduction of CK activity in cultured neurons (Aksenov et al., 1998, 2000; David et al., 1998). Consistent with these findings, creatine accumulates in old transgenic mice expressing a mutant APP and in the hippocampus of AD patients (Gallant et al., 2006). Our finding that the partial Df of HPD ortholog promoted the accumulation of oligomeric A $\beta 42$ provides a likely explanation for the worsening of age-dependent geotaxis performance. Yet, such degree of $A \beta$ accumulation seems to be sufficient to impact negatively upon neuronal function without inducing gross neuropathological changes up to 18 d.p.e. With regard to possible mechanisms for $A \beta$ accretion in the context of lower HPPD expression, the reduction in CK activity as a consequence of high tyrosine levels may accelerate $\mathrm{A} \beta$ aggregation or impair its clearance due to lower ATP availability and oxidative stress (Meyer et al., 2006). Moreover, A $\beta 42$ oligomers induce oxidative stress (Butterfield et al., 2013) leading to a vicious cycle in disease progression. Alternatively, the possibility that a partial Df of
HPPD is more directly involved in A $\beta$ accumulation deserves further investigation. Inhibitors of HPPD such as nitisinone are used to treat patients with hereditary Tyrosinemia type 1 in which downstream metabolites of HPPD activity accumulate and are highly toxic to the kidney and liver (Mayorandan et al., 2014; Zeybek et al., 2015). Long-term outcome of patients under nitisinone treatment show a high frequency of progressive cognitive impairment that has been related with chronically elevated tyrosine levels (Masurel-Paulet et al., 2008; Thimm et al., 2012). Early reports on tyrosine levels in the cerebrospinal fluid of $\mathrm{AD}$ as compared with controls remain controversial (Degrell et al., 1989; Martinez et al., 1993) and there are no studies on the levels and/or activity of HPPD in AD. In light of our results regarding $A \beta$ accumulation, such studies may be relevant to better understand the complex pathogenesis of $\mathrm{AD}$.

In summary, our work describes the first genetic screen to search for modifiers of wild-type $A \beta 42$ neurotoxicity in the CNS of Drosophila by exploring age-dependent alterations in a complex behavior. So far, this strategy has led us to identify candidate genes that warrant further research to determine their significance in sporadic AD. 


\section{AUTHOR CONTRIBUTIONS}

LFB-C, MSM and NIB performed the experiments, analyzed results, drafted and revised the manuscript; MFC and LM designed the work, analyzed data, interpreted the results and revised the manuscript. EMC designed the work, analyzed data, interpreted the results and wrote the article.

\section{FUNDING}

This work was supported by grants from the Alzheimer's Association (IIRG 11-205127 to EMC), Agencia Nacional de Promoción Científica y Tecnológica (ANPCyT) PICT2013-0318

\section{REFERENCES}

Aksenov, M., Aksenova, M., Butterfield, D. A., and Markesbery, W. R. (2000). Oxidative modification of creatine kinase BB in Alzheimer's disease brain. J. Neurochem. 74, 2520-2527. doi: 10.1046/j.1471-4159.2000.0742520.x

Aksenov, M. Y., Aksenova, M. V., Markesbery, W. R., and Butterfield, D. A. (1998). Amyloid $\beta$-peptide (1-40)-mediated oxidative stress in cultured hippocampal neurons. Protein carbonyl formation, $\mathrm{CK}$ BB expression and the level of $\mathrm{Cu}$, Zn and Mn SOD mRNA. J. Mol. Neurosci. 10, 181-192. doi: 10.1007/bf027 61773

Attrill, H., Falls, K., Goodman, J. L., Millburn, G. H., Antonazzo, G., Rey, A. J., et al. (2016). FlyBase: establishing a gene group resource for Drosophila melanogaster. Nucl. Acids Res. 44, D786-D792. doi: 10.1093/nar/gkv1046

Bennett, D. A., Schneider, J. A., Arvanitakis, Z., Kelly, J. F., Aggarwal, N. T., Shah, R. C., et al. (2006). Neuropathology of older persons without cognitive impairment from two community-based studies. Neurology 66, 1837-1844. doi: 10.1212/01.WNL.0000219668.47116.e6

Bouleau, S., and Tricoire, H. (2015). Drosophila models of Alzheimer's disease: advances, limits and perspectives. J. Alzheimers Dis. 45, 1015-1038. doi: 10.3233/JAD-142802

Brownlee, J. M., Heinz, B., Bates, J., and Moran, G. R. (2010). Product analysis and inhibition studies of a causative Asn to Ser variant of 4-hydroxyphenylpyruvate dioxygenase suggest a simple route to the treatment of Hawkinsinuria. Biochemistry 49, 7218-7226. doi: 10.1021/bi1008112

Butterfield, D. A., Swomley, A. M., and Sultana, R. (2013). Amyloid $\beta$-peptide (1-42)-induced oxidative stress in Alzheimer disease: importance in disease pathogenesis and progression. Antioxid. Redox Signal. 19, 823-835. doi: $10.1089 /$ ars.2012.5027

Cacace, R., Sleegers, K., and Broeckhoven, C. V. (2016). Molecular genetics of early-onset alzheimer disease revisited. Alzheimers Dement. 12, 733-748. doi: 10.1016/j.jalz.2016.01.012

Campion, D., Flaman, J. M., Brice, A., Hannequin, D., Dubois, B., Martin, C., et al. (1995). Mutations of the presenilin I gene in families with earlyonset alzheimer's disease. Hum. Mol. Genet. 4, 2373-2377. doi: 10.1093/hmg/4. 12.2373

Cao, W., Song, H.-J., Gangi, T., Kelkar, A., Antani, I., Garza, D., et al. (2008). Identification of novel genes that modify phenotypes induced by Alzheimer's $\beta$-amyloid overexpression in Drosophila. Genetics 178, 1457-1471. doi: 10.1534/genetics.107.078394

Cook, K. (2016). Bloomington Drosophila Stock Center at Indiana University. Bloomington: Indiana University. Available online at: http://flystocks.bio. indiana.edu/Browse/df/dfkit.php. [Accessed on May 13, 2016].

Cook, R. K., Christensen, S. J., Deal, J. A., Coburn, R. A., Deal, M. E., Gresens, J. M., et al. (2012). The generation of chromosomal deletions to provide extensive coverage and subdivision of the Drosophila melanogaster genome. Genome Biol. 13:R21. doi: 10.1186/gb-2012-13-3-r21

Crowther, D. C., Kinghorn, K. J., Miranda, E., Page, R., Curry, J. A., Duthie, F. A. I., et al. (2005). Intraneuronal $A \beta$, non-amyloid aggregates and neurodegeneration in a Drosophila model of Alzheimer's disease. Neuroscience 132, 123-135. doi: 10.1016/j.neuroscience.2004.12.025 (to EMC), PICT2013-1382 (to MFC) and CONICET-PIP0378 (to LM).

\section{ACKNOWLEDGMENTS}

We thank Cecilia Rotondaro, Agata Fernández-Gamba and Luciana Pujol Lereis for their contributions to this work. A $\beta 42$ transgenic fly was kindly provided by Dr. Mary Konsolaki (Rutgers University). Bloomington Drosophila Stock Center and the Vienna Drosophila RNAi Center provided the flies stocks. LFB-C fellowship is supported by ANPCyT; MSM and NIB fellowships are supported by CONICET. MFC, LM and EMC are Principal Investigators supported by CONICET.

David, S., Shoemaker, M., and Haley, B. E. (1998). Abnormal properties of creatine kinase in Alzheimer's disease brain: correlation of reduced enzyme activity and active site photolabeling with aberrant cytosol-membrane partitioning. Brain. Res. Mol. 54, 276-287. doi: 10.1016/s0169-328x(97) 00343-4

de Andrade, R. B., Gemelli, T., Rojas, D. B., Funchal, C., Dutra-Filho, C. S., Wannmacher, C. M., et al. (2012). Tyrosine impairs enzymes of energy metabolism in cerebral cortex of rats. Mol. Cell. Biochem. 364, 253-261. doi: 10.1007/s11010-012-1225-y

De Strooper, B., and Karran, E. (2016). The cellular phase of Alzheimer's disease. Cell. 164, 603-615. doi: 10.1016/j.cell.2015.12.056

Degrell, I., Hellsing, K., Nagy, E., and Niklasson, F. (1989). Amino acid concentrations in cerebrospinal fluid in presenile and senile dementia of Alzheimer type and multi-infarct dementia. Arch. Gerontol. Geriatr. 9, 123-135. doi: 10.1016/0167-4943(89)90033-2

Fernandez-Funez, P., de Mena, L., and Rincon-Limas, D. E. (2015). Modeling the complex pathology of Alzheimer's disease in Drosophila. Exp. Neurol. 274, 58-71. doi: 10.1016/j.expneurol.2015.05.013

Ferreira, G. K., Carvalho-Silva, M., Gomes, L. M., Scaini, G., Teixeira, L. J., Mota, I. T., et al. (2015). The characterization of neuroenergetic effects of chronic L-tyrosine administration in young rats: evidence for striatal susceptibility. Metab. Brain Dis. 30, 215-221. doi: 10.1007/s11011-0149615-3

Ferreira, G. K., Carvalho-Silva, M., Gonçalves, C. L., Vieira, J. S., Scaini, G. Ghedim, F. V., et al. (2012). L-tyrosine administration increases acetylcholinesterase activity in rats. Neurochem. Int. 61, 1370-1374. doi: 10.1016/j.neuint.2012.09.017

Finelli, A., Kelkar, A., Song, H.-J., Yang, H., and Konsolaki, M. (2004). A model for studying Alzheimer's A 342 -induced toxicity in Drosophila melanogaster. Mol. Cell. Neurosci. 26, 365-375. doi: 10.1016/j.mcn.2004.03.001

Gallant, M., Rak, M., Szeghalmi, A., Del Bigio, M. R., Westaway, D., Yang, J., et al. (2006). Focally elevated creatine detected in amyloid precursor protein (APP) transgenic mice and Alzheimer disease brain tissue. J. Biol. Chem. 281, 5-8. doi: 10.1074/jbc.C500244200

Gargano, J. W., Martin, I., Bhandari, P., and Grotewiel, M. S. (2005). Rapid iterative negative geotaxis (RING): a new method for assessing age-related locomotor decline in Drosophila. Exp. Gerontol. 40, 386-395. doi: 10.1016/j. exger.2005.02.005

Hatakeyama, S., Yada, M., Matsumoto, M., Ishida, N., and Nakayama, K. I. (2001). $\mathrm{U}$ box proteins as a new family of ubiquitin-protein ligases. J. Biol. Chem. 276, 33111-33120. doi: 10.1074/jbc.m102755200

Hegele, A., Kamburov, A., Grossmann, A., Sourlis, C., Wowro, S., Weimann, M. et al. (2012). Dynamic protein-protein interaction wiring of the human spliceosome. Mol. Cell 45, 567-580. doi: 10.1016/j.molcel.2011. 12.034

Jackson, G. R. (2008). Guide to understanding Drosophila models of neurodegenerative diseases. PLoS Biol. 6:e53. doi: 10.1371/journal.pbio. 0060053

Kandimalla, R. J. L., Prabhakar, S., Binukumar, B. K., Wani, W. Y., Gupta, N., Sharma, D. R., et al. (2011). Apo-E4 allele in conjunction with A $\beta 42$ and tau 
in CSF: biomarker for Alzheimers disease. Curr. Alzheimer Res. 8, 187-196. doi: 10.2174/156720511795256071

Kandimalla, R. J. L., Wani, W. Y., Binukumar, B. K., and Gill, K. D. (2012). siRNA against presenilin 1 (PS1) down regulates amyloid $\beta 42$ production in IMR-32 cells. J. Biomed. Sci. 19:2. doi: 10.1186/1423-0127-19-2

Karran, E., Mercken, M., and De Strooper, B. (2011). The amyloid cascade hypothesis for Alzheimer's disease: an appraisal for the development of therapeutics. Nat. Rev. Drug Discov. 10, 698-712. doi: 10.1038/nrd3505

Khan, S., and Bloom, G. S. (2016). Tau: the center of a signaling nexus in Alzheimer's disease. Front. Neurosci. 10:31. doi: 10.3389/fnins.2016.00031

Lenz, S., Karsten, P., Schulz, J. B., and Voigt, A. (2013). Drosophila as a screening tool to study human neurodegenerative diseases. J. Neurochem. 127, 453-460. doi: $10.1111 /$ jnc. 12446

Lim, J. Y., Ott, S., and Crowther, D. C. (2016). Drosophila melanogaster as a model for studies on the early stages of Alzheimer's disease. Methods Mol. Biol. 1303, 227-239. doi: 10.1007/978-1-4939-2627-5_13

Liu, H., Han, M., Li, Q., Zhang, X., Wang, W. A., and Huang, F. D. (2015). Automated rapid iterative negative geotaxis assay and its use in a genetic screen for modifiers of A 342 -induced locomotor decline in Drosophila. Neurosci. Bull. 31, 541-549. doi: 10.1007/s12264-014-1526-0

Maarouf, C. L., Daugs, I. D., Kokjohn, T. A., Walker, D. G., Hunter, J. M., Kruchowsky, J. C., et al. (2011). Alzheimer's disease and non-demented high pathology control nonagenarians: comparing and contrasting the biochemistry of cognitively successful aging. PLoS One 6:e27291. doi: 10.1371/journal.pone. 0027291

Martinez, M., Frank, A., Diez-Tejedor, E., and Hernanz, A. (1993). Amino acid concentrations in cerebrospinal fluid and serum in Alzheimer's disease and vascular dementia. J. Neural Transm. Park. Dis. Dement. Sect. 6, 1-9. doi: $10.1007 /$ bf02252617

Masurel-Paulet, A., Poggi-Bach, J., Rolland, M. O., Bernard, O., Guffon, N., Dobbelaere, D., et al. (2008). NTBC treatment in tyrosinaemia type I: long-term outcome in French patients. J. Inherit. Metab. Dis. 31, 81-87. doi: 10.1007/s10545-008-0793-1

Mayorandan, S., Meyer, U., Gokcay, G., Segarra, N. G., de Baulny, H. O., van Spronsen, F., et al. (2014). Cross-sectional study of 168 patients with hepatorenal tyrosinaemia and implications for clinical practice. Orphanet. J. Rare Dis. 9:107. doi: 10.1186/s13023-014-0107-7

Meyer, L. E., Machado, L. B., Santiago, A. P., da-Silva, W. S., De Felice, F. G., Holub, O., et al. (2006). Mitochondrial creatine kinase activity prevents reactive oxygen species generation: antioxidant role of mitochondrial kinase-dependent ADP re-cycling activity. J. Biol. Chem. 281, 37361-37371. doi: 10.1074/jbc. m604123200

Moloney, A., Sattelle, D. B., Lomas, D. A., and Crowther, D. C. (2010). Alzheimer's disease: insights from Drosophila melanogaster models. Trends Biochem. Sci. 35, 228-235. doi: 10.1016/j.tibs.2009.11.004

Moran, M. T., Tare, M., Kango-Singh, M., and Singh, C. (2013). Homeotic gene teashirt (tsh) has a neuroprotective function in amyloid- $\beta 42$ mediated neurodegeneration. PLoS One 8:e80829. doi: 10.1371/journal.pone.0080829

Newman, M., Musgrave, I. F., Musgrave, F. I., and Lardelli, M. (2007). Alzheimer disease: amyloidogenesis, the presenilins and animal models. Biochim. Biophys. Acta 1772, 285-297. doi: 10.1016/j.bbadis.2006.12.001

Perez-Nievas, B. G., Stein, T. D., Tai, H. C., Dols-Icardo, O., Scotton, T. C., Barroeta-Espar, I., et al. (2013). Dissecting phenotypic traits linked to human resilience to Alzheimer's pathology. Brain 136, 2510-2526. doi: 10.1093/brain/awt171

Prüßing, K., Voigt, A., and Schulz, J. B. (2013). Drosophila melanogaster as a model organism for Alzheimer's disease. Mol. Neurodegener. 35, 1-11. doi: $10.1186 / 1750-1326-8-35$
Pushkarsky, T., Yurchenko, V., Vanpouille, C., Brichacek, B., Vaisman, I., Hatakeyama, S., et al. (2005). Cell surface expression of CD147/EMMPRIN is regulated by cyclophilin 60 . J. Biol. Chem. 280, 27866-27871. doi: 10.1074/jbc. M503770200

Rival, T., Page, R. M., Chandraratna, D. S., Sendall, T. J., Ryder, E., Liu, B., et al. (2009). Fenton chemistry and oxidative stress mediate the toxicity of the $\beta$-amyloid peptide in a Drosophila model of Alzheimer's disease. Eur. J. Neurosci. 29, 1335-1347. doi: 10.1111/j.1460-9568.2009. 06701.x

Sarantseva, S., Timoshenko, S., Bolshakova, O., Karaseva, E., Rodin, D., Schwarzman, A. L., et al. (2009). Apolipoprotein E-mimetics inhibit neurodegeneration and restore cognitive functions in a transgenic Drosophila model of Alzheimer's disease. PLoS One 4:e8191. doi: 10.1371/journal.pone. 0008191

Scheltens, P., Blennow, K., Breteler, M. M. B., de Strooper, B., Frisoni, G. B., Salloway, S., et al. (2016). Alzheimer's disease. Lancet 338, 505-517. doi: 10.1016/s0140-6736(15)01124-1

Scott, C. R. (2006). The genetic tyrosinemias. Am. J. Med. Genet. C Semin. Med. Genet. 142C, 121-126. doi: 10.1007/978-1-4614-6430-3_279-1

Shulman, J. M., Imboywa, S., Giagtzoglou, N., Powers, M. P., Hu, Y., Devenport, D., et al. (2014). Functional screening in Drosophila identifies Alzheimer's disease susceptibility genes and implicates Tau-mediated mechanisms. Hum. Mol. Genet. 23, 870-877. doi: 10.1093/hmg/ ddt 478

Skalsky, Y. M., Ajuh, P. M., Parker, C., Lamond, A. I., Goodwin, G., and Cooper, C. S. (2001). PRCC, the commonest TFE3 fusion partner in papillary renal carcinoma is associated with pre-mRNA splicing factors. Oncogene. 20, 178-187. doi: 10.1038/sj.onc.1204056

Steffener, J., and Stern, Y. (2012). Exploring the neural basis of cognitive reserve in aging. Biochim. Biophys. Acta 1822, 467-473. doi: 10.1016/j.bbadis.2011. 09.012

Tan, L., Schedl, P., Song, H.-J., Garza, D., and Konsolaki, M. (2008). The toll $\rightarrow \mathrm{NFkB}$ signaling pathway mediates the neuropathological effects of the human Alzheimer's A 342 Polypeptide in Drosophila. PLoS One. 3:e3966. doi: 10.1371/journal.pone.0003966

Thimm, E., Richter-Werkle, R., Kamp, G., Molke, B., Herebian, D., Klee, D., et al. (2012). Neurocognitive outcome in patients with hypertyrosinemia type I after long-term treatment with NTBC. J. Inherit. Metab. Dis. 35, 263-268. doi: 10.1007/s10545-011-9394-5

Wallimann, T., Tokarska-Schlattner, M., and Schlattner, U. (2011). The creatine kinase system and pleiotropic effects of creatine. Amino Acids 40, 1271-1296. doi: 10.1007/s00726-011-0877-3

Zeybek, A. C., Kiykim, E., Soyucen, E., Cansever, S., Altay, S., Zubarioglu, T., et al. (2015). Hereditary tyrosinemia type 1 in Turkey: twenty year single-center experience. Pediatr. Int. 57, 281-289. doi: 10.1111/ped. 12503

Conflict of Interest Statement: The authors declare that the research was conducted in the absence of any commercial or financial relationships that could be construed as a potential conflict of interest.

Copyright (๑) 2017 Belfiori-Carrasco, Marcora, Bocai, Ceriani, Morelli and Castaño. This is an open-access article distributed under the terms of the Creative Commons Attribution License (CC BY). The use, distribution and reproduction in other forums is permitted, provided the original author(s) or licensor are credited and that the original publication in this journal is cited, in accordance with accepted academic practice. No use, distribution or reproduction is permitted which does not comply with these terms. 